\title{
Evaluation of Salivary Biomarkers for the Diagnosis of Periodontitis
}

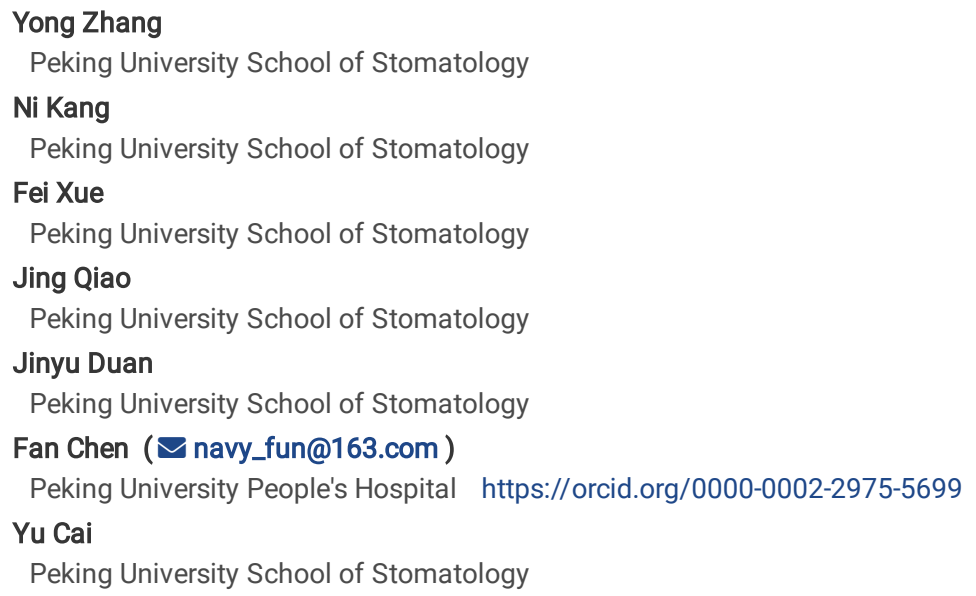

Version of Record: A version of this preprint was published at BMC Oral Health on May 17th, 2021. See the published version at https://doi.org/10.1186/s12903-021-01600-5. 


\section{Abstract}

Background: To investigate the diagnostic potential of salivary biomarkers and built a prediction panel for diagnosing periodontal disease.

Methods: In a cross-sectional study, a total of 85 participants were enrolled in the study and divided into healthy, gingivitis and periodontitis groups based on their periodontal examine results, a full mouth periodontal examination were received and unstimulated saliva were collected. Salivary IL-1 $\beta$, MMP-8, ICTP and $\mathrm{Pg}$ were assessed and their potential of diagnosing periodontal disease were analyzed, the combinational prediction panels of periodontal disease were evaluated.

Results: The periodontitis group showed significantly higher level of IL-1 $\beta$, MMP-8, ICTP and Pg compared to healthy group and higher level of IL-1 $\beta$, ICTP and $\mathrm{Pg}$ compared to gingivitis group, the gingivitis group showed higher level of IL-1 $\beta$, MMP-8 and Pg compared to healthy group. As a single marker, IL-1 $\beta$ showed the best diagnostic value of four markers and exhibited an AUC value of 0.88 with $94 \%$ sensitivity and $71 \%$ specificity to discriminate periodontitis from healthy subjects, an AUC value of 0.81 with $82 \%$ sensitivity and $76 \%$ specificity to discriminate gingivitis from healthy subjects and an AUC value of 0.67 with $69 \%$ sensitivity and $64 \%$ specificity to discriminate periodontitis from gingivitis subjects. The combination of IL-1, ICTP and Pg exhibited the highest value to discriminate periodontitis from healthy (AUC $=0.94)$ and gingivitis (AUC=0.78) subjects, the combination of IL-1 and MMP-8 exhibited the best value to discriminate gingivitis from healthy subjects (AUC=0.85).

Conclusions: Salivary IL-1 $\beta$, MMP-8, ICTP and Pg showed discriminate efficiency for diagnosing periodontal disease, the combination of IL-1, ICTP and Pg can be used to discriminate periodontitis from healthy and gingivitis subjects and the combination of IL-1 and MMP-8 can be used to discriminate gingivitis from healthy subjects.

\section{Background}

Periodontal disease is a chronic inflammatory disease induced by pathogenic bacteria and resulted in connective tissue and alveolar bone destructions. Traditionally diagnosis of periodontal disease is based on clinical and radiographic examinations that reflect a previous history of disease but lack of reflecting disease activity. Early detection of periodontal tissue destruction can be useful for us to monitor disease's progression and prevent future destruction. Saliva is a simple noninvasive body fluid with omics constituents that can reflects the physiologic status of the periodontal tissue[1].

In the past decades, various of salivary markers have been found to be related to the periodontal disease, bacteria[2], host enzymes[3], cytokines[4] and bone metabolic products[5] have been investigated to be different between periodontitis and healthy subjects, however there were inconsistent or even contrary results in other studies[6-8]. As we know, during the progress of periodontal disease, in the gingivitis stage, patients just perform gingival inflammation and without connective tissue or bone destruction, then in the early stage of periodontitis, patients perform gingival inflammation with connective tissue destruction and then alveolar bone destruction at the later stage of disease. We believe that the change of different markers occur no simultaneously but consecutively at different phase of periodontal disease, a biomarker combination can be more effectively used for diagnosing disease. Salivary interleukin (IL)-1 [3] ], matrix metalloproteinase (MMP)-8[10], pyridinoline cross-linked carboxyterminal telopeptide of type I collagen (ICTP)[11] and Porphyromonas gingivalis $(\mathrm{Pg})[12]$ were high prevalence in the populations and revealed a high relationship of periodontitis, the aim of this study is to evaluate discriminate efficiency of these markers among healthy, gingivitis and chronic periodontitis patients and pair them to build a prediction panel for diagnosing periodontal disease.

\section{Methods}

1. Study design: This study was designed and performed as a cross sectional study. It was approved by the human subjects ethics board of Peking University School and Hospital of Stomatology and was conducted in accordance with the Helsinki Declaration of 1975, as revised in 2013. All participants were informed verbally and in writing and their written informed consent were received. The inclusion criteria were: 1) 20-65 years of age. 2) at least had 20 teeth. 3) underwent no medical treatment during the last 3 months before examination and sampling. 4) no smoker. 5) no history of systemic diseases. The exclusion criteria were 1) wore orthodontic appliances. 2) were pregnant or currently at the breast-feeding period. 3) undergone periodontal therapy within 6 months before the examination and sampling .

2. Patients: All participants were recruited at the department of periodontology, first clinical division, Peking University School and Hospital of Stomatology. All participants were divided into 3 groups determined by their clinical periodontal examine results: healthy group (H), gingivitis group (G) and periodontitis group (P) according to the Centers for Disease Control and Prevention/America Academy of Periodontology (CDC/AAP) definition.

1) Healthy group $(\mathrm{H})$ : Subjects with absence of bleeding on probing (BOP $<10 \%)$, probing depth $(\mathrm{PD}) \leq 3 \mathrm{~mm}$, no clinical attachment loss $(\mathrm{CAL})$, no radiographic bone loss, no sign of other inflammatory lesions in the oral mucosa.

2) Gingivitis group (G): Subjects with presence of bleeding on probing in at least 10 sites and $B O P \geq 10 \%$, PD $\leq 4 \mathrm{~mm}$, no clinical attachment loss, no radiographic bone loss.

3) Periodontitis group $(P)$ : Subjects with presence of bleeding on probing in at least 10 sites and $B O P \geq 10 \%$, $\geq 2$ sites with $P D \geq 5$ mm (not on the same tooth) and $\geq 2$ sites $C A L \geq 5 \mathrm{~mm}$ (not on the same tooth).

3. Clinical evaluations: All participants received a full mouth periodontal examination and a medical and dental history evaluation. All permanent teeth were taken with a 10-mm manual periodontal probe (PCP10-SE, Hu-Friedy, Chicago, USA) and rounded off upwards to the nearest millimeter. Plaque Index (PLI) and 
BOP were measured at buccal and lingual surface of all teeth, PD and CAL were measure at 6 sites of all teeth. BOP was present if the probed site bled about 20 seconds after probing the buccal and lingual surfaces of each tooth.

4. Saliva collection and analysis: Participants were asked to refrain from eating, drinking, smoking or oral hygiene procedures for at least two hours prior to the saliva collection. Unstimulated saliva samples were collected and saliva samples were centrifuged at $5000 \mathrm{~g}$ for 5 min at $4^{\circ} \mathrm{C}$. Supernatants were removed from the pellet, aliquots of the resultant supernatant and pellets were stored at $-80^{\circ} \mathrm{C}$ until analysis.

Salivary IL-1 $\beta$, MMP-8 and ICTP levels were detected and measured using commercial enzyme-linked immunosorbent assay (ELISA) kits obtained from R\&D systems (Minneapolis, MN, USA) and Orion Diagnostica (Espoo, Finland) according to the manufacturer's instructions. Salivary P. gingivalis (Pg) DNA was extracted from pellets using the UltraClean Microbial DNA Isolation Kit (MO BIO Laboratories Inc, Carlsbad, California, USA), then quantities of Pg were determined by quantitative real time PCR (qPCR), primers for the 16S rRNA gene of Pg were below: forward primer:5'-GCGCTCAACGTTCAGCC-3'; reverse primer:5'-CACGAATTCCGCCTGC-3'. qPCR was performed in duplicate in reaction volumes of 10 $\mu$ l using power SYBR-Green Master Mix (Applied Biosystems, Foster City, California, USA) for $15 \mathrm{~min}$ at $95.8 \mathrm{C}$ for initial denaturing, followed by 40 cycles of $95.8^{\circ} \mathrm{C}$ for $30 \mathrm{~s}$ and $60.8^{\circ} \mathrm{C}$ for $30 \mathrm{~s}$, and then Cycle Threshold (Ct) value were calculated.

\section{Statistical analysis}

Based on the results from Mishra et al.[11] and Wu et al.[13], a minimum of 25 participants per group was set as the sample size of the present study. The SPSS statistical program (Version 21.0; SPSS Inc., Chiacago, IL, USA) was used to analyses the data. The mean with standard deviation was used to describe the variables of the demographic and clinical characteristics and the distribution of biomarkers among healthy, gingivitis and periodontitis groups. Independent $t$ tests were used to evaluate the differences among three groups. The correlations between biomarkers and clinical indexes were assessed using the linear regression analysis, and logistic regression adjusted for age and sex was used to establish panels for predicting gingivitis and periodontitis according to an automatic stepwise selection strategy. The receiver operating characteristics (ROC) curve analysis and corresponding area under the curve (AUC) analysis were used to evaluate the performance of biomarkers and predictive panels. The sensitivity and specificity for the biomarker combinations were estimated by identifying the cut-off point of the predicted probability that yielded the highest sum of sensitivity and specificity. Statistical significance was defined as $\mathrm{p}<0.05$.

\section{Results}

A total of 85 participants were enrolled in this study, the demographic and clinical characteristics of all participants are presented in Table 1. The demographics of mean age and tooth were balanced among three groups. 25 patients were in healthy group, 28 patients were in gingivitis group and 32 were in periodontitis group. As anticipated, compared to the healthy group, the gingivitis and periodontitis groups showed higher PLI and BOP, the periodontitis group showed older, higher PD and CAL compared to healthy and gingivitis groups.

The salivary level of IL-1 $\beta$, MMP-8, ICTP and Pg were measured and are shown in Table 1. ANOVA analysis was performed to investigate difference among three groups, the periodontitis group showed significantly higher level of IL-1 $\beta$, MMP-8, ICTP and Pg compared to healthy group and higher level of IL-1 $\beta$, ICTP and Pg compared to gingivitis group, the gingivitis group showed higher level of IL-1 $\beta$, MMP-8 and Pg compared to healthy group. The salivary MMP-8 showed no difference between periodontitis and gingivitis groups and the ICTP showed no difference between gingivitis and healthy groups.

Table 1

Characteristics of study participants among healthy, gingivitis and periodontitis groups

\begin{tabular}{|c|c|c|c|}
\hline & Healthy group $(\mathrm{N}=25)$ & Gingivitis group $(\mathrm{N}=\mathbf{2 8})$ & Periodontitis group $(\mathrm{N}=32)$ \\
\hline Age & $24.68 \pm 3.52$ & $26.18 \pm 3.84$ & $42.75 \pm 5.74$ ** \#\# \\
\hline Gender (M/F) & $(12 / 13)$ & $(12 / 16)$ & $(18 / 14)$ \\
\hline Tooth & $27.7 \pm 1.1$ & $27.6 \pm 1.1$ & $27.4 \pm 0.9$ \\
\hline Plaque index (PLI) & $0.22 \pm 0.28$ & $0.72 \pm 0.64$ * & $1.34 \pm 0.77 * \#$ \\
\hline Bleeding on probing (BOP, \%) & $1.1 \pm 1.8$ & $20.5 \pm 10.6$ ** & $65.7 \pm 17.2$ **\#\# \\
\hline \multicolumn{4}{|l|}{$(\mathrm{PD}, \mathrm{mm})$} \\
\hline Clinical attachment loss (CAL, mm) & 0 & 0 & $4.92 \pm 0.52$ ** \#\# \\
\hline $\mathrm{IL}-1 \beta(\mathrm{pg} / \mathrm{ml})$ & $92.2 \pm 31.9$ & $138.9 \pm 45.8$ ** & $166.2 \pm 61.6$ ** \# \\
\hline MMP-8 (ng/ml) & $435.8 \pm 180.6$ & $591.8 \pm 235.1$ * & $663.9 \pm 276.3$ ** \\
\hline ICTP (pg/ml) & $528.8 \pm 141.1$ & $620.4 \pm 206.1$ & $793.4 \pm 243.7$ ** \#\# \\
\hline $\mathrm{Pg}$ (Ct value) & $13.78 \pm 1.23$ & $14.52 \pm 0.95$ * & $15.14 \pm 1.21 * * \#$ \\
\hline \multicolumn{4}{|c|}{ *: significant different compared to healthy group $(*: p<0.05 * *: p<0.01)$} \\
\hline \multicolumn{4}{|c|}{ \#: significant different compared to gingivitis group (\#:p<0.05 \#\#:p<0.01) } \\
\hline
\end{tabular}


We next evaluated the correlation between each marker and clinical indexes (PD and BOP) using linear regression model, the results showed IL-1 $\beta$ and ICTP were moderate correlated with PD $(r=0.48,0.46, p<0.01)$ and BOP $(r=0.42,0.48, p<0.01), P g$ was moderate correlated with PD $(r=0.41, p<0.01)$ and mild correlated with BOP $(r=0.36, p<0.01)$, MMP-8 was mild correlated with PD $(r=0.32, p<0.01)$ and BOP $(r=0.36, p<0.01)($ Table 2$)$.

Table 2

Correlations analysis between markers and clinical characteristics of study population (Sperman correlation coefficients)

\begin{tabular}{|lllll|}
\hline & PD & \multicolumn{3}{c|}{ BOP } \\
\cline { 2 - 5 } & Spearman's $\boldsymbol{p}$ & p value & Spearman's $\boldsymbol{p}$ & p value \\
\hline IL-1 $\beta$ & 0.475 & $<0.001$ & 0.422 & $<0.001$ \\
MMP-8 & 0.319 & 0.007 & 0.352 & 0.001 \\
\hline ICTP & 0.459 & $<0.001$ & 0.479 & $<0.001$ \\
\hline Pg & 0.412 & 0.003 & 0.356 & 0.001 \\
\hline
\end{tabular}

1) Periodontits vs. Healthy groups

All of four markers we tested in this study showed significant difference between periodontitis and healthy groups (Table 1 ). IL-1 $\beta$ yielded an AUC value of 0.88 , and other three markers yielded AUC values from 0.75 to 0.85 (Table 3). Logistic regression was used to evaluate different combinations of four biomarkers, among the different combinations, IL-1 $\beta$ and ICTP in combination yielded higher AUC value of 0.92 with $91 \%$ sensitivity and $88 \%$ specificity and the combination of IL-1 13 , ICTP and MMP-8 yielded an AUC value of 0.93 with $94 \%$ sensitivity and $81 \%$ specificity, the combination of IL-1 3 , ICTP and Pg yielded an AUC value of 0.94 with $94 \%$ sensitivity and $83 \%$ specificity, and four biomarkers combined together yielded the best AUC value of 0.95 (Table 4 ).

Table 3

Performance of salivary biomarkers of study population among healthy, gingivitis and periodontitis groups

\begin{tabular}{|c|c|c|c|c|c|c|c|c|c|c|c|c|}
\hline & \multicolumn{4}{|c|}{ Periodontitis vs. Health (P vs. H) } & \multicolumn{4}{|c|}{ Gingivitis vs. Health (G vs. H) } & \multicolumn{4}{|c|}{ Peridoontitis vs. Gingivitis (P vs. G) } \\
\hline & sensitivity & specificity & $\begin{array}{l}\text { AUC value } \\
\text { (95\% } \\
\text { confidence } \\
\text { intervals) }\end{array}$ & $\begin{array}{l}\mathrm{p} \\
\text { value }\end{array}$ & sensitivity & specificity & $\begin{array}{l}\text { AUC value } \\
\text { (95\% } \\
\text { confidence } \\
\text { intervals) }\end{array}$ & $\begin{array}{l}\mathrm{p} \\
\text { value }\end{array}$ & sensitivity & specificity & $\begin{array}{l}\text { AUC value } \\
\text { ( } 95 \% \\
\text { confidence } \\
\text { intervals) }\end{array}$ & $\begin{array}{l}\mathrm{p} \\
\text { valut }\end{array}$ \\
\hline IL-1 $\beta$ & 0.94 & 0.71 & $\begin{array}{l}0.879 \\
(0.792- \\
0.966)\end{array}$ & $\begin{array}{l}< \\
0.001\end{array}$ & 0.821 & 0.760 & $\begin{array}{l}0.810 \\
(0.685- \\
0.935)\end{array}$ & $\begin{array}{l}< \\
0.001\end{array}$ & 0.688 & 0.643 & $\begin{array}{l}0.673 \\
(0.536- \\
0.823)\end{array}$ & 0.02 \\
\hline $\begin{array}{l}\text { MMP- } \\
8\end{array}$ & 0.65 & 0.84 & $\begin{array}{l}0.755 \\
(0.626- \\
0.884)\end{array}$ & 0.001 & 0.679 & 0.840 & $\begin{array}{l}0.753 \\
(0.617- \\
0.889)\end{array}$ & 0.002 & 0.563 & 0.536 & $\begin{array}{l}0.560 \\
(0.413- \\
0.706)\end{array}$ & $0.42 i$ \\
\hline ICTP & 0.81 & 0.88 & $\begin{array}{l}0.845 \\
(0.735- \\
9.955)\end{array}$ & $\hat{0}_{0.001}$ & 0.643 & 0.600 & $\begin{array}{l}0.632 \\
(0.481- \\
0.783)\end{array}$ & 0.199 & 0.781 & 0.679 & $\begin{array}{l}0.727 \\
(0.593- \\
0.860)\end{array}$ & 0.00 \\
\hline $\mathrm{Pg}$ & 0.88 & 0.56 & $\begin{array}{l}0.777 \\
(0.655- \\
0.898)\end{array}$ & $\begin{array}{l}< \\
0.001\end{array}$ & 0.714 & 0.680 & $\begin{array}{l}0.691 \\
(0.539- \\
0.842)\end{array}$ & 0.017 & 0.709 & 0.689 & $\begin{array}{l}0.685 \\
(0.543- \\
0.827)\end{array}$ & 0.01 \\
\hline
\end{tabular}

Table 4

Performance of salivary biomarker combinations to discriminate periodontitis from healthy subjects.

\begin{tabular}{|llll|}
\hline & sensitivity & specificity & AUC value (95\% confidence intervals) \\
\hline IL-1 $\beta+$ MMP-8 & 0.906 & 0.800 & $0.886(0.799-0.973)$ \\
\hline IL-1 $\beta+P g$ & 0.906 & 0.813 & $0.910(0.828-0.992)$ \\
\hline IL-1 $\beta+$ MMP-8 + Pg & 0.906 & 0.840 & $0.921(0.845-0.998)$ \\
\hline ICTP + MMP-8 & 0.844 & 0.800 & $0.856(0.755-0.958)$ \\
\hline ICTP + Pg & 0.813 & 0.800 & $0.879(0.790-0.968)$ \\
IL-1 $\beta+$ ICTP & 0.906 & 0.875 & $0.920(0.845-0.995)$ \\
\hline IL-1 $\beta+$ ICTP + MMP-8 & 0.938 & 0.813 & $0.931(0.869-0.993)$ \\
\hline IL-1 $\beta+$ ICTP + Pg & 0.938 & 0.830 & $0.935(0.874-0.996)$ \\
\hline IL-1 $\beta+$ ICTP + MMP-8 + Pg & 0.938 & 0.844 & $0.948(0.896-0.999)$ \\
\hline
\end{tabular}

2) Gingivitis vs. Healthy groups 
IL-1 $\beta$, MMP-8 and Pg levels showed significant differences between gingivitis and healthy groups (Table 1), IL-1 3 and MMP-8 yielded similar AUC values, Pg yielded lower AUC value compared to other two markers (Table 3). After logistic regression analysis, among the different combinations, IL-1 $\beta$ and MMP-8 in combination yielded an AUC value of 0.85 with $93 \%$ sensitivity and $68 \%$ specificity, and the combination of IL-1 $\beta$, MMP-8 and Pg yielded the best AUC value of 0.86 with $86 \%$ sensitivity and $80 \%$ specificity (Table 5 ).

Table 5

Performance of salivary biomarker combinations to discriminate gingivitis from healthy subjects

\begin{tabular}{|llll|}
\hline & sensitivity & specificity & AUC value (95\% confidence intervals) \\
\hline IL-1 $\beta+$ MMP-8 & 0.929 & 0.680 & $0.846(0.737-0.955)$ \\
\hline $\mathrm{IL}-1 \beta+\mathrm{Pg}$ & 0.821 & 0.760 & $0.824(0.709-0.940)$ \\
\hline $\mathrm{IL}-1 \beta+\mathrm{MMP}-8+\mathrm{Pg}$ & 0.857 & 0.800 & $0.857(0.752-0.962)$ \\
\hline $\mathrm{IL}-1 \beta+\mathrm{MMP}-8+\mathrm{Pg}+\mathrm{ICTP}$ & 0.893 & 0.760 & $0.860(0.757-0.963)$ \\
\hline
\end{tabular}

3) Periodontitis vs. Gingivitis groups

IL-1 $\beta$, ICPT and Pg levels showed significant differences between periodontitis and gingivitis groups and yielded similar AUC values (Table 1, 3). After logistic regression analysis, among different combinations of two markers, the combination of IL-1 $\beta$ and ICTP yielded a higher AUC value of 0.76 with $78 \%$ sensitivity and $71 \%$ specificity, IL-1 $\beta$ and Pg yielded similar AUC value, and the combination of IL-1 $\beta, P g$ and ICTP yielded the best AUC value of 0.78 with $81 \%$ sensitivity and $75 \%$ specificity (Table 6).

Table 6

Performance of salivary biomarker combinations to discriminate periodontitis from gingivitis subjects

\begin{tabular}{|llll|}
\hline & sensitivity & specificity & AUC value (95\% confidence intervals) \\
\hline IL-1 $\beta+$ ICTP & 0.781 & 0.714 & $0.753(0.619-0.883)$ \\
\hline IL-1 $\beta+P g$ & 0.719 & 0.717 & $0.735(0.598-0.858)$ \\
\hline ICTP + Pg & 0.781 & 0.707 & $0.730(0.603-0.857)$ \\
\hline IL-1 $\beta+$ ICTP + Pg & 0.813 & 0.750 & $0.779(0.655-0.900)$ \\
\hline IL-1 $\beta+$ ICTP + MMP-8 + Pg & 0.781 & 0.786 & $0.779(0.656-0.902)$ \\
\hline
\end{tabular}

\section{Discussion}

Periodontal disease has been diagnosed by clinical performance of BOP, PD, CAL and radiographic evidence of alveolar bone loss. Although their utility is reliable, but still costly and need clinician's professional experience. Saliva have been proved to be a high potential tool in early diagnosis and monitoring oral and systemic diseases[14, 15], and in the previous studies, a number of salivary markers have been demonstrated to be different between diseased and healthy controls, but up to now there is no clear and convinced biomarker that can be used for diagnosing periodontal disease.

We need a balance between microbial and host response to keep periodontal health, during the progress of periodontal disease, the balance were broken and bacterial invasion, host inflammatory response, tissue and bone destructions non-simultaneously occurred. After bacteria (Pg) invasion, markers of inflammation (IL-1 $\beta$ ) are released[16], enzymes such as MMP-8 are produced and activated by host cells leading to the degradation of connective tissue[17], and bone degradation resulted in releasing ICTP into periodontal tissues and saliva[5]. In the present study we selected these four markers (IL-1 $\beta$, MMP-8, ICTP, $\mathrm{Pg})$, evaluated their diagnostic efficiency of gingivitis and periodontitis and built a valuable prediction panel using their combinations.

To our knowledge, IL-1 $\beta$ is a well-known inflammatory stimulator that is discriminative between healthy and periodontal lesions[4]; Pg is significantly associated with periodontal disease and has been used as a potential screening biomarker of periodontitis[18]. In this study, both of IL-1 $\beta$ and Pg showed significant difference among three groups, their salivary level were increasing in the gingivitis group and more higher in the periodontitis group. Our data were in consistence with other studies[12,19], and besides significant elevation in gingivitis and periodontitis groups, our data also demonstrated positively association of IL-1 $\beta$ and Pg with clinical indexes (PD and BOP), showed they indeed reflected the periodontal status and can be valuable candidates to predict periodontal disease.

Recent investigations showed MMP-8 to be an indicator especially for early periodontitis[20, 21]. In the current study, MMP-8 in the gingivitis and periodontitis groups showed significant higher compared to the healthy group, but there was no significant difference between periodontitis and gingivitis group. As we know, MMP-8 is one of the most prevalence host proteinases and is correlated with periodontal inflammatory destruction[17]. Since our participants' gingiva already expressed inflammation in the gingivitis and periodontitis groups, they presented higher level of MMP-8. The main difference between gingivitis and periodontitis groups in the present study was bone loss, there seems to be no relationship between MMP-8 and bone loss and resulted in no continue increasing of MMP-8 in the periodontitis group compared to the gingivitis group. The present results were in accordance with Morelli's study[22], salivary MMP-8 exhibited significant difference between gingivitis and healthy groups, but no significant difference between periodontitis and gingivitis groups. Nascimento's study also showed MMP-8 significantly increased during the process of experimental gingivitis[23], all these data indicated that MMP-8 increased with the development of gingival inflammation and kept stable in the subsequent stage of disease. 
Before an effective diagnosis of periodontitis, considerable amount of alveolar bone destruction would have established, when we measured the damage of bone loss clinically, a 2 to $3 \mathrm{~mm}$ threshold changes are needed before it exhibiting obvious destruction, that may delay the diagnosis and treatment[24]. As a breakdown product of Type I collagen, ICTP is the major constituent of alveolar bone and was considered to reflect alveolar bone degradation and periodontal disease activity[25]. Not surprising in our study, ICTP was found to be no significant difference between gingivitis and healthy groups as there was no bone loss in these two groups. Apparent alveolar bone loss in the periodontitis group resulted in their ICTP level were significant higher compared to gingivitis and healthy groups, which is in accordance with Mishra[11] and Giannobile's[26] studies and they concluded that increased ICTP can be used to differentiate active gingivitis from periodontitis, and Payne's[27] results also stated that salivary ICTP concentrations is significantly associated with alveolar bone height loss.

After confirming their difference among periodontitis, gingivitis and healthy groups, we examined their ability to discriminate different periodontal clinical phenotypes. As a single marker, IL-1 $\beta$ showed the best diagnostic value of these four candidates: exhibited an AUC value of 0.88 with $94 \%$ sensitivity and $71 \%$ specificity to discriminate periodontitis from healthy subjects, an AUC value of 0.81 with $82 \%$ sensitivity and $76 \%$ specificity to discriminate gingivitis from healthy subjects and an AUC value of 0.67 with $69 \%$ sensitivity and $64 \%$ specificity to discriminate periodontitis from gingivitis subjects, this is valuable results and is consistent with data from Jaedicke's[4] and Nazar's[28] investigations, they concluded that IL-1 $\beta$ is the most robust salivary biomarkers for periodontal disease. Hassan[29]'s results exhibited there was a positive relationship between salivary IL-1 $\beta$ and the gingival inflammation during pregnancy. These results supported us including IL-1 $\beta$ as a predictive overall indicator of gingivitis and periodontitis.

Different markers may be peaked at different course of disease and when biomarkers of host and microbe origin are combined, the detection of periodontitis maybe improved rather than when used individually[13, 30, 31], previous studies revealed stronger discriminatory power when IL-1 $\beta$, MMP-8 and other markers were combined compared with single analysis[32], Pg and MMP-8 in combination[33], ICTP and MMP-8 in combination[34] also exhibited more predictive values. Our data showed that IL-1 $\beta$ individually revealed an AUC value of 0.88 to discriminate periodontitis from healthy subjects, the combination of IL-1 $\beta$, MMP-8 and Pg strong performance improvement to an AUC value of 0.92, that is consistent with Gursoy's results[35, 36], they calculated IL-1 $\beta$, MMP-8 and Pg together to obtain a cumulative risk score that is highly related with advanced periodontitis. Although showed that biomarker combinations facilitated more robust prediction of periodontal progression and stability, our results were different from Gursoy's results and demonstrated that IL-1 $\beta$ and ICTP in combination yielded a similar AUC value (0.920) to differentiate periodontitis from healthy subjects compared with the combination of IL-1 $\beta$, MMP-8 and Pg (0.921), indicating that IL-1 $\beta$ and ICTP were more valuable for predicting periodontitis and the combination of IL-1 $\beta$, ICTP and Pg exhibited the best AUC value (0.95) to discriminate periodontitis from healthy subjects .

In addition, as a nondestructive and reversible gingival inflammation stage, we enrolled gingivitis subjects into our study and assigned participants with more homogeneous clinical phenotypes. Our results showed that MMP-8 in the periodontitis group was not significantly elevated compared to gingivitis group, and ICTP in the gingivitis group was not significantly elevated compared to healthy group, indicating that for predicting the different status, different marker combinations should be used to achieve an effective diagnosing power. After logistic regression analysis, the combination of IL-1, ICTP and Pg not only yielded the best AUC value to discriminate periodontitis from healthy subjects, but also exhibited the best performance to discriminate the periodontitis from gingivitis subjects $(A U C=0.78$ ). To discriminate gingivitis from healthy subjects, although IL-1 $3, \mathrm{MMP}-8$ and Pg together revealed the best AUC value of 0.86 , IL-1 $\beta$ and MMP-8 in combination yielded a slight lower AUC value of 0.85 , these AUC values were lower to the combination of discriminating the periodontitis from healthy subjects (IL-1, ICTP and Pg, AUC = 0.94), but still were all above acceptable AUC value of $0.75[37$ ] and could be potentially used for the clinical diagnosis.

\section{Conclusion}

In conclusion, the results of our study showed that there were significant differences of salivary IL-1, MMP-8, ICTP and Pg among healthy, gingivitis and periodontitis groups, meanwhile the efficiency of prediction panels for diagnosing gingivitis and periodontitis were revealed, the combination of IL-1, ICTP and $\mathrm{Pg}$ can be used to discriminate periodontitis from healthy and gingivitis subjects, the combination of IL-1 and MMP-8 can be used to discriminate gingivitis from healthy subjects. However some limitations still need to be addressed: larger study sample's size, longitudinal studies such as experiment gingivitis or periodontitis designs and more precise categories designs are needed to make more convinced evaluation of our selected salivary biomarkers in the future.

\section{Abbreviations}

IL-1ß:1 interleukin (IL)-1ß; MMP-8: matrix metalloproteinase-8; ICTP: pyridinoline cross-linked carboxyterminal telopeptide of type I collagen; Pg:

Porphyromonas gingivalis; BOP: bleeding on probing; PD: probing depth; CAL: clinical attachment loss; qPCR: quantitative real time polymerase chain reaction; ROC: receiver operating characteristics; AUC: area under the curve.

\section{Declarations}

Funding: The study was supported by National Natural Science Foundations of China (Project Number: 81800978) and Peking University School and Hospital of Stomatology Cao Caifang Gengyun Foundation (2017).

Availability of data and materials: The datasets generated during the current study are available from the corresponding author on reasonable request

Authors' Contributions: Y.Z, F.C. and Y.C. wrote and edited the manuscript. Y.Z, F.C. and Y.C. conceptualized the overall strategy and developed the clinical translation and implementation. Y.Z, F.X, J.Q. and JY.D enrolled patients to the protocol, and implemented the saliva collection and processing. Y.Z, N.K and F.C. designed and performed the biomarkers measurement. Y.Z and F.C designed and performed the statistical analyses. F.C. and Y.C. were the study's principal investigators. All authors read and approved the final manuscript.

Page 6/8 
Ethics approval and consent to participate The Ethics Committee of the Peking University School and Hospital of Stomatology approved the study protocol. All procedures performed in studies involving human participants were in accordance with the ethical standards of the institutional and/or national research committee and with the 1964 Helsinki Declaration and its later amendments or comparable ethical standards. Written informed consent for participation was obtained from each subject recruited in this study.

Consent for publication This manuscript was not involved with details, images, or videos relating to individual participants. Written informed consent of this research was obtained from all the subjects involved

Competing interests The authors declare that they have no conflict of interest.

\section{References}

1. Fuentes L, Yakob M, Wong DT. Emerging horizons of salivary diagnostics for periodontal disease. Br Dent J. 2014;217(10):567-73.

2. Belstrom D, Sembler-Moller ML, Grande MA, Kirkby N, Cotton SL, Paster BJ, Twetman S, Holmstrup P. Impact of Oral Hygiene Discontinuation on Supragingival and Salivary Microbiomes. JDR Clin Trans Res. 2018;3(1):57-64.

3. Ramseier CA, Kinney JS, Herr AE, Braun T, Sugai JV, Shelburne CA, Rayburn LA, Tran HM, Singh AK, Giannobile WV. Identification of pathogen and hostresponse markers correlated with periodontal disease. J Periodontol. 2009;80(3):436-46.

4. Jaedicke KM, Preshaw PM, Taylor JJ. Salivary cytokines as biomarkers of periodontal diseases. Periodontol 2000. 2016;70(1):164-83.

5. Frodge BD, Ebersole JL, Kryscio RJ, Thomas MV, Miller CS. Bone remodeling biomarkers of periodontal disease in saliva. J Periodontol. 2008;79(10):1913-9.

6. Syndergaard B, Al-Sabbagh M, Kryscio RJ, Xi J, Ding X, Ebersole JL, Miller CS. Salivary biomarkers associated with gingivitis and response to therapy. J Periodontol. 2014;85(8):e295-303.

7. Belstrom D, Damgaard C, Kononen E, Gursoy M, Holmstrup P, Gursoy UK. Salivary cytokine levels in early gingival inflammation. J Oral Microbiol. 2017;9(1):1364101.

8. Teles RP, Likhari V, Socransky SS, Haffajee AD. Salivary cytokine levels in subjects with chronic periodontitis and in periodontally healthy individuals: a cross-sectional study. J Periodontal Res. 2009;44(3):411-7.

9. Gursoy UK, Kononen E, Uitto VJ, Pussinen PJ, Hyvarinen K, Suominen-Taipale L, Knuuttila M. Salivary interleukin-1 beta concentration and the presence of multiple pathogens in periodontitis. J Clin Periodontol. 2009;36(11):922-7.

10. de Morais EF, Pinheiro JC, Leite RB, Santos PPA, Barboza CAG, Freitas RA. Matrix metalloproteinase-8 levels in periodontal disease patients: A systematic review. J Periodontal Res. 2018;53(2):156-63.

11. Mishra D, Gopalakrishnan S, Arun KV, Kumar TS, Devanathan S, Misra SR. Evaluation of Salivary Levels of Pyridinoline Cross Linked Carboxyterminal Telopeptide of Type I Collagen (ICTP) in Periodontal Health and Disease. J Clin Diagn Res. 2015;9(9):ZC50-55.

12. Damgaard C, Danielsen AK, Enevold C, Massarenti L, Nielsen CH, Holmstrup P, Belstrom D. Porphyromonas gingivalis in saliva associates with chronic and aggressive periodontitis. J Oral Microbiol. 2019;11(1):1653123.

13. Wu YC, Ning L, Tu YK, Huang CP, Huang NT, Chen YF, Chang PC. Salivary biomarker combination prediction model for the diagnosis of periodontitis in a Taiwanese population. J Formos Med Assoc. 2018;117(9):841-8.

14. Dawes C, Wong DTW. Role of Saliva and Salivary Diagnostics in the Advancement of Oral Health. J Dent Res. 2019;98(2):133-41.

15. Zhang Y, Sun J, Lin CC, Abemayor E, Wang MB, Wong DT. The emerging landscape of salivary diagnostics. Periodontol 2000. 2016;70(1):38-52.

16. Loos BG, Tjoa S. Host-derived diagnostic markers for periodontitis: do they exist in gingival crevice fluid? Periodontol 2000 2005, 39:53-72.

17. Sorsa T, Gursoy UK, Nwhator S, Hernandez M, Tervahartiala T, Leppilahti J, Gursoy M, Kononen E, Emingil G, Pussinen PJ, et al. Analysis of matrix metalloproteinases, especially MMP-8, in gingival creviclular fluid, mouthrinse and saliva for monitoring periodontal diseases. Periodontol 2000. 2016;70(1):142-63.

18. Liljestrand JM, Gursoy UK, Hyvarinen K, Sorsa T, Suominen AL, Kononen E, Pussinen PJ. Combining salivary pathogen and serum antibody levels improves their diagnostic ability in detection of periodontitis. J Periodontol. 2014;85(1):123-31.

19. Sexton WM, Lin Y, Kryscio RJ, Dawson DR 3rd, Ebersole JL, Miller CS. Salivary biomarkers of periodontal disease in response to treatment. J Clin Periodontol. 2011;38(5):434-41.

20. Heikkinen AM, Raisanen IT, Tervahartiala T, Sorsa T. Cross-sectional analysis of risk factors for subclinical periodontitis; active matrix metalloproteinase-8 as a potential indicator in initial periodontitis in adolescents. J Periodontol. 2019;90(5):484-92.

21. Schmalz G, Hubscher AE, Angermann H, Schmidt J, Schmickler J, Legler TJ, Ziebolz D. Associations of chairside salivary aMMP-8 findings with periodontal parameters, potentially periodontal pathogenic bacteria and selected blood parameters in systemically healthy adults. Diagn Microbiol Infect Dis. 2019;95(2):179-84.

22. Morelli T, Stella M, Barros SP, Marchesan JT, Moss KL, Kim SJ, Yu N, Aspiras MB, Ward M, Offenbacher S. Salivary biomarkers in a biofilm overgrowth model. J Periodontol. 2014;85(12):1770-8.

23. Nascimento GG, Baelum V, Sorsa T, Tervahartiala T, Skottrup PD, Lopez R. Salivary levels of MPO, MMP-8 and TIMP-1 are associated with gingival inflammation response patterns during experimental gingivitis. Cytokine. 2019;115:135-41.

24. Kinney JS, Ramseier CA, Giannobile WV. Oral fluid-based biomarkers of alveolar bone loss in periodontitis. Ann N Y Acad Sci. 2007;1098:230-51.

25. Taba M Jr, Kinney J, Kim AS, Giannobile WV. Diagnostic biomarkers for oral and periodontal diseases. Dent Clin North Am. 2005;49(3):551-71. vi. 
26. Giannobile WV, Lynch SE, Denmark RG, Paquette DW, Fiorellini JP, Williams RC. Crevicular fluid osteocalcin and pyridinoline cross-linked carboxyterminal telopeptide of type I collagen (ICTP) as markers of rapid bone turnover in periodontitis. A pilot study in beagle dogs. J Clin Periodontol. 1995;22(12):90310.

27. Payne JB, Stoner JA, Lee HM, Nummikoski PV, Reinhardt RA, Golub LM. Serum bone biomarkers and oral/systemic bone loss in humans. J Dent Res. 2011;90(6):747-51

28. Nazar Majeed Z, Philip K, Alabsi AM, Pushparajan S, Swaminathan D. Identification of Gingival Crevicular Fluid Sampling, Analytical Methods, and Oral Biomarkers for the Diagnosis and Monitoring of Periodontal Diseases: A Systematic Review. Dis Markers. 2016;2016:1804727.

29. Hassan MN, Belibasakis GN, Gumus P, Ozturk VO, Emingil G, Bostanci N. Annexin-1 as a salivary biomarker for gingivitis during pregnancy. J Periodontol. 2018;89(7):875-82.

30. Lundmark A, Hu YOO, Huss M, Johannsen G, Andersson AF, Yucel-Lindberg T. Identification of Salivary Microbiota and Its Association With Host Inflammatory Mediators in Periodontitis. Front Cell Infect Microbiol. 2019;9:216.

31. Kc S, Wang XZ, Gallagher JE. Diagnostic sensitivity and specificity of host-derived salivary biomarkers in periodontal disease amongst adults: Systematic review. J Clin Periodontol. 2020;47(3):289-308.

32. Ebersole JL, Nagarajan R, Akers D, Miller CS. Targeted salivary biomarkers for discrimination of periodontal health and disease(s). Front Cell Infect Microbiol. 2015;5:62.

33. Salminen A, Gursoy UK, Paju S, Hyvarinen K, Mantyla P, Buhlin K, Kononen E, Nieminen MS, Sorsa T, Sinisalo J, et al. Salivary biomarkers of bacterial burden, inflammatory response, and tissue destruction in periodontitis. J Clin Periodontol. 2014;41(5):442-50.

34. Gursoy UK, Kononen E, Pradhan-Palikhe P, Tervahartiala T, Pussinen PJ, Suominen-Taipale L, Sorsa T. Salivary MMP-8, TIMP-1, and ICTP as markers of advanced periodontitis. J Clin Periodontol. 2010;37(6):487-93.

35. Gursoy UK, Kononen E, Pussinen PJ, Tervahartiala T, Hyvarinen K, Suominen AL, Uitto VJ, Paju S, Sorsa T. Use of host- and bacteria-derived salivary markers in detection of periodontitis: a cumulative approach. Dis Markers. 2011;30(6):299-305.

36. Gursoy UK, Pussinen PJ, Salomaa V, Syrjalainen S, Kononen E. Cumulative use of salivary markers with an adaptive design improves detection of periodontal disease over fixed biomarker thresholds. Acta Odontol Scand. 2018;76(7):493-6.

37. Hosmer DW, Lemeshow S: Applied Logistic Regression, Second Edition. New York: Wiley-Interscience Publication; 2000. 